\title{
Axonal Growth Is Sensitive to the Levels of Katanin, a Protein That Severs Microtubules
}

\author{
Arzu Karabay, ${ }^{1,2}$ Wenqian Yu, ${ }^{1}$ Joanna M. Solowska, ${ }^{1}$ Douglas H. Baird, ${ }^{1}$ and Peter W. Baas ${ }^{1}$ \\ ${ }^{1}$ Department of Neurobiology and Anatomy, Drexel University College of Medicine, Philadelphia, Pennsylvania 19129, and ${ }^{2}$ Department of Molecular \\ Biology and Genetics, Istanbul Technical University, 34469 Istanbul, Turkey
}

\begin{abstract}
Katanin is a heterodimeric enzyme that severs microtubules from the centrosome so that they can move into the axon. Katanin is broadly distributed in the neuron, and therefore presumably also severs microtubules elsewhere. Such severing would generate multiple short microtubules from longer microtubules, resulting in more microtubule ends available for assembly and interaction with other structures. In addition, shorter microtubules are thought to move more rapidly and undergo organizational changes more readily than longer microtubules. In dividing cells, the levels of P60-katanin (the subunit with severing properties) increase as the cell transitions from interphase to mitosis. This suggests that katanin is regulated in part by its absolute levels, given that katanin activity is high during mitosis. In the rodent brain, neurons vary significantly in katanin levels, depending on their developmental stage. Levels are high during rapid phases of axonal growth but diminish as axons reach their targets. Similarly, in neuronal cultures, katanin levels are high when axons are allowed to grow avidly but drop when the axons are presented with target cells that cause them to stop growing. Expression of a dominant-negative P60-katanin construct in cultured neurons inhibits microtubule severing and is deleterious to axonal growth. Overexpression of wild-type P60-katanin results in excess microtubule severing and is also deleterious to axonal growth, but this only occurs in some neurons. Other neurons are relatively unaffected by overexpression. Collectively, these observations indicate that axonal growth is sensitive to the levels of P60-katanin, but that other factors contribute to modulating this sensitivity.
\end{abstract}

Key words: katanin; microtubule; axon; neuron; neuronal development; axonal growth

\section{Introduction}

Neurons are terminally postmitotic cells that use their microtubules not for the formation of a mitotic spindle but rather for the elaboration of an elongated axon. Changes in the configuration and behavior of microtubules within specific regions of the neuron underlie important events in axonal differentiation such as elongation, retraction, navigation, branching, and sprouting (Baas and Buster, 2004). Although the dynamics (assembly and disassembly) of microtubules can account for much of their behavior, other mechanisms are crucial as well. Studies from our laboratory suggest that forces generated by motor proteins are essential for transporting microtubules into the axon, and potentially for integrating microtubules with neighboring microtubules and other cytoskeletal elements (Ahmad et al., 1998, 2000). Recent observations strongly suggest that the capacity of a microtubule to move in response to these forces is intimately related to the length of the microtubule (Dent et al., 1999; Wang and Brown, 2002), with shorter microtubules moving more avidly than longer microtubules. Thus a critical means for regulating

Received Feb. 2, 2004; revised May 10, 2004; accepted May 11, 2004.

This work was supported by two grants from the National Institutes of Health (P.W.B.) and a State of Pennsylvania Tobacco Formula Funds grant (D.H.B.). We thank Dr. Francis J. McNally for helpful discussions and for providing human katanin constructs. We also thank Dr. Gianluca Gallo for helpful discussions at the beginning of these studies and two outstanding reviewers for their helpful comments.

Correspondence should be addressed to Dr. Peter W. Baas, Drexel University College of Medicine, Department of Neurobiology and Anatomy, 2900 West Queen Lane, Philadelphia, PA 19129. E-mail: Peter.W.Baas@drexel.edu. DOI:10.1523/JNEUROSCI.1382-04.2004

Copyright $\odot 2004$ Society for Neuroscience $\quad$ 0270-6474/04/245778-11\$15.00/0 microtubule movement may be to regulate microtubule length. Given that neuronal microtubules are not capped at their plus ends and can be very stable along much of their length (Baas and Black, 1990), it seems unlikely that neurons generate short microtubules in strategic locations exclusively via disassembly of longer polymers. Several lines of evidence now indicate that long microtubules can be fractured into shorter microtubules by a process known as microtubule severing. Studies from our laboratory have shown evidence for microtubule severing in the cell body of the neuron (Ahmad et al., 1999), at sites of axonal branch formation (Yu et al., 1994), and within growth cones (Dent et al., 1999).

Microtubule severing appears to be induced primarily by katanin, an enzyme that hydrolyzes ATP to make breaks in the lattice of the polymer (Quarmby and Lohret, 1999). We have shown that katanin is abundant in cultured neurons, widely distributed, and functionally important for severing microtubules from the centrosome so that they can move into the developing axon (Ahmad et al., 1999). The fact that katanin is present in all regions of the neuron suggests that it also severs microtubules elsewhere, not just at the centrosome. In addition, the widespread distribution of katanin suggests that it is locally activated and deactivated rather than, or in addition to, being recruited to sites where microtubules need to be severed. For these reasons, most efforts have focused on the potential mechanisms that regulate whether katanin can break a microtubule that it encounters (McNally et al., 2002). For example, in neurons, a variety of 
microtubule-associated proteins (MAPs) might regulate katanin by interfering with its access to the microtubule lattice. In the present study, we sought to determine whether there is also tight regulation of the levels of katanin in the neuron during axonal growth and whether fluctuations in these levels can affect the disposition of the microtubule array and, in turn, modulate the growth of the axon.

\section{Materials and Methods}

P60-katanin sequencing and preparation of probes. Reverse transcriptasePCR was used to obtain the complete sequence of P60-katanin from newborn rat whole-brain tissues. Total RNA was obtained after tissue disruption with Tri Reagent, according to the manufacturer's instructions (Sigma, St. Louis, MO), and isopropanol precipitation. cDNA was generated from total RNA using an oligo- $\mathrm{dT}_{(15)}$ primer and Powerscript reverse transcriptase (Clontech, Palo Alto, CA) according to the manufacturer's instructions. PCR primer sequences were based primarily on available mouse and human P60-katanin sequences. Alignments identified regions of identical or highly similar nucleotide sequences that were selected as annealing sites for primers. PCR products were ligated into TA cloning vectors [Qiagen (Valencia, CA) and Invitrogen (Carlsbad, CA)] and then sequenced on an ABI Prism DNA Sequencer 377 (Applied Biosystems International, Foster City, CA). Independent clones were sequenced to provide at least six readings of the cDNA of rat P60-katanin. Based on the rat P60-katanin clone, we prepared two constructs for expression in cultured neurons and fibroblasts. One of these, termed "wild-type," is the intact P60-katanin sequence coupled to green fluorescent protein (GFP), whereas the other, termed "dominant-negative," is precisely the same except that it has a single amino acid substitution in the ATP-binding domain that renders the molecule incapable of severing microtubules (McNally et al., 2000, 2002).

Predicting possible solubility problems related to the bacterial expression of P60-katanin (Hartman et al., 1998), we produced anti-peptide antibodies. The peptides used for the generation of these antibodies were based on sequences that avoided the conserved C-terminal domain (which is common in other AAA proteins). Anti-P60-katanin synthetic peptide antibodies were generated by immunization of rabbits at Zymed Laboratories (San Francisco, CA). A particularly good antibody was produced against a region corresponding to amino acids 115-148, and this antibody was used for the present studies.

Sense and antisense riboprobes were synthesized with a digoxigenin (DIG) RNA labeling kit (Boehringer Mannheim, Mannheim, Germany). Template for the production of nonradioactive riboprobes for in situ hybridization was made by subcloning the full-length P60 into pBluescript SK-plasmid (Stratagene, La Jolla, CA). After template linearization, sense and antisense digoxigenin-labeled riboprobes were transcribed with a ribonucleotide mix containing digoxigenin UTP (Roche, Basel, Switzerland) and either T3 or T7 RNA polymerase (Promega, Madison, WI), according to the manufacturer's instructions.

Western blotting. Rat brain tissue, a rat fibroblastic cell line termed RFL6, and a human kidney cell line termed 293 were used for Western blotting with the anti-P60-katanin antibody. The human kidney cells were induced to express the rat GFP-katanin wild-type construct before blotting. Cell extracts and tissue homogenates were mixed with Laemmli sample buffer. SDS-PAGE and Western blotting were performed according to established procedures (Sambrook et al., 1989). Blots were blocked with $5 \%$ nonfat milk in TTBS (50 mM Tris, $150 \mathrm{~mm} \mathrm{NaCl}$, and $0.1 \%$ Tween 20), incubated with synthetic anti-P60 antibody diluted 1:20,000 in TTBS with 5\% milk, washed, and reacted with goat anti-rabbit IgG conjugated with HRP (Jackson ImmunoResearch, West Grove, PA). Bound secondary antibody was detected with a chemiluminescent peroxidase substrate (Super Signal; Pierce, New York, NY).

Immunolabeling and in situ hybridization of brain sections. Sections of mouse brain at various stages of development were obtained from a cryostat and prepared for single-label visualization of P60-katanin immunoreactivity using a standard peroxidase technique (Solowska et al., 2002). Brains from at least three mice at each stage were completely sectioned and immunolabeled. Sections were treated with $0.2 \% \mathrm{H}_{2} \mathrm{O}_{2}$ in
TBST (10 mm Tris-HCl, pH 7.5, $150 \mathrm{~mm} \mathrm{NaCl}$, and 0.1\% Tween 20), blocked with $5 \%$ skim milk in TBS, incubated overnight with anti-P60katanin antibody, and then exposed to anti-rabbit secondary antibody conjugated with HRP (Jackson ImmunoResearch). HRP activity was detected using $0.5 \mathrm{mg} / \mathrm{ml}$ diaminobenzidine, $0.006 \% \mathrm{H}_{2} \mathrm{O}_{2}$, and $0.1 \%$ nickel ammonium sulfate in $50 \mathrm{~mm}$ Tris, $\mathrm{pH}$ 7.5. For in situ hybridization, frozen sections $(16-20 \mu \mathrm{m})$ were collected and in situ hybridized for $21 \mathrm{hr}$ at $55^{\circ} \mathrm{C}$ in a humid chamber, using $200 \mathrm{ng} / \mathrm{ml}$ DIG-labeled riboprobes in hybridization buffer containing 50\% formamide and $10 \%$ dextran sulfate. Sections were then washed in $2 \times \mathrm{SSC}$ at $65^{\circ} \mathrm{C}$ for $1 \mathrm{hr}$ and in $0.1 \times \mathrm{SSC}$ at $65^{\circ} \mathrm{C}$ for $1 \mathrm{hr}$. Hybrids were detected using anti-DIG antibody conjugated with alkaline phosphatase (Boehringer Mannheim). Labeled sections were imaged using a 135TV inverted microscope (Carl Zeiss, Oberkochen, Germany) equipped with a high-resolution CCD (NU200; Photometrics, Tucson, AZ).

Preparation, transfection, and immunolabeling of cell cultures. For explants of basilar pontine neurons, the basilar pontine nuclei (BPN) were removed by excising the superficial swellings just caudal to the cephalic flexure. Microexplants $\sim 200-300 \mu \mathrm{m}$ in diameter were dissected from the BPN and cultured as a source of axons that develop in situ as mossy fibers (Hatten et al., 1998; Solowska et al., 2002). Reaggregates of cerebellar cells were prepared as target cells for BPN axons. Cell-culture reagents were purchased from Invitrogen (Gaithersburg, MD) unless otherwise indicated. Briefly, cerebella were dissected from two postnatal rats on day 4 and dissociated using trypsin, DNase (Worthington, Lake Wood, NJ), and trituration as described previously (Hatten et al., 1998). Dissociated cerebellar cells were allowed to reaggregate during overnight incubation at $37^{\circ} \mathrm{C}$ and $5 \% \mathrm{CO}_{2}$ in a $15 \mathrm{ml}$ tube and suspended in $10 \mathrm{ml}$ of MEM supplemented with $10 \%$ fetal calf serum (Hyclone, Logan, UT), $10 \%$ horse serum, $0.1 \%$ glucose, $200 \mu \mathrm{M}$ L-glutamine, penicillin-streptomycin $(200 \mathrm{U} / \mathrm{ml}$ each), and N2 supplement. After $24 \mathrm{hr}$, the cells formed reaggregates that were plated onto coverslip-bottomed culture wells (Baird et al., 1994) coated with $25 \mu \mathrm{g} / \mathrm{ml} \mathrm{laminin}$. After the reaggregates adhered to the coverslips, the serum-containing medium was replaced with medium that lacked serum but was otherwise identical. BPN explants were then added to the reaggregates. Cultures of rat superior cervical sympathetic ganglia neurons were prepared as described previously (He and Baas, 2003).

For transfection of the P60-katanin constructs, the single-cell suspension of sympathetic neurons was resuspended in Nucleofector solution (Amaxa Biosystems, Cologne, Germany), and neurons were then transfected with GFP alone (control), GFP-P60-katanin wild-type, or GFPP60-katanin dominant-negative using an Amaxa Biosystems electroporator nucleofection device according to the manufacturer's instructions and recommended settings. After transfection, neurons were transferred to $37^{\circ} \mathrm{C} \mathrm{N} 2$ medium (He and Baas, 2003), plated onto poly-D-lysinetreated glass coverslips overnight, and then exposed to laminin for $8 \mathrm{hr}$. Using this approach, transfection efficiency was generally $5 \%$, with GFP fluorescence appearing within the first several hours after transfection. In some experiments, neurons were plated at high density (as a monolayer) in plastic culture dishes for $2 \mathrm{~d}$, after which the cultures were treated with trypsin to release the neurons, which were then replated on a polylysinelaminin substrate for $8 \mathrm{hr}$ ( $\mathrm{He}$ and Baas, 2003).

For immunofluorescence studies on microtubule distribution, cultures were washed briefly with $37^{\circ} \mathrm{C}$ PBS and then simultaneously fixed and extracted with $0.2 \%$ glutaraldehyde and $0.2 \%$ Triton X-100 for 15 min. Cultures were washed three times with PBS and $0.1 \%$ Tween 20 for $5 \mathrm{~min}$, quenched three times with $10 \mathrm{mg} / \mathrm{ml}$ sodium borohydride for 10 $\mathrm{min}$, and then blocked with $10 \%$ normal goat serum and $10 \mathrm{mg} / \mathrm{ml} \mathrm{BSA}$ in PBS and $0.1 \%$ Tween 20 for $1 \mathrm{hr}$. After blocking, cultures were exposed to an anti-GFP polyclonal antibody (1:100; Abcam, Cambridge, UK) for $1 \mathrm{hr}$ at $37^{\circ} \mathrm{C}$. (Enhancement of GFP fluorescence by immunofluorescence assisted in discerning between the cells expressing the GFP-P60katanin constructs and those that were not expressing, given that the fluorescence of the GFP itself tended to diminish during the fixation and extraction.) Cultures were then rinsed three times for 5 min in PBS, followed by exposure to an anti- $\beta$-tubulin antibody directly conjugated to Cy3 (1:100; Sigma) together with FITC-conjugated secondary goat anti-rabbit antibody (1:100; Jackson ImmunoResearch). Cells were then 
rinsed extensively in PBS and mounted in a medium that reduces photobleaching. Images were obtained using a Pascal confocal microscope (Carl Zeiss). For immunofluorescence studies on P60-katanin levels, the cultures were double-labeled by initial exposure to a monoclonal antibody to GFP (1:100; Sigma), together with our P60-katanin polyclonal antibody (1: 1000) for $1 \mathrm{hr}$, and then by exposure to FITCconjugated goat anti-mouse and rhodamineconjugated goat anti-rabbit secondary antibodies for $1 \mathrm{hr}$ (both at 1:100; Jackson ImmunoResearch). Images were obtained on an Axiovert 200 microscope (Carl Zeiss) that was equipped with a high-resolution CCD (Orca ER; Hamamatsu, Shizouka, Japan).

Immunofluorescence was quantified in some of the experiments in which cells were immunostained with the polyclonal P60-katanin antibody or one of the GFP antibodies. For these experiments, all images were obtained using identical camera, microscope, and imaging criteria (such as gain, brightness and contrast, and exposure time). Efforts were made to ensure that the images were not saturated and that minimal bleaching of the fluorescence occurred before image acquisition. Digital gray values of image pixels representing arbitrary fluorescence units (AFUs) were obtained using the public domain NIH Image Program (developed at the National Institutes of Health). For the studies on cultured basilar pontine neurons, the gray value of axon segments $10 \mu \mathrm{m}$ in length was determined by averaging the brightest gray value of pixels within the axon (minus the gray value of the neighboring substrate) in three lines perpendicular to the axon, one line at each end of the segment, and one line in the center of the segment. AFUs were computed in this manner for segments located at or near the distal tips or growth cones of axons and in axon segments located 35-100 $\mu \mathrm{m}$ more proximal within the same axon.

For studies on cultured sympathetic neurons, fluorescence intensity was quantified for neurons that had not been induced to express any katanin construct, neurons that had been induced to express GFP alone, neurons that had been induced to express the wild-type GFP-P60katanin construct, and neurons that had been induced to express the dominant-negative GFP-P60-katanin construct. For cultured RFL6 cells, the cells were categorized as either mitotic or interphase, and fluorescence intensity was determined. For sympathetic neurons, quantification was performed on the cell body. For RFL6 cells, quantification was performed on the entire cell. In each case, AFUs for all pixels were summed.

\section{Results}

\section{Rat P60-katanin sequence and probes}

In our previous study on katanin inneurons, we used polyclonal antibodies against the human katanin sequences (Ahmad et al., 1999). As part of a long-term commitment to studying katanin in the developing rodent nervous system, we have now sequenced P60-katanin from rat brain. As expected, there is a great deal of similarity among the human (90\% homology), mouse (92\% homology), and Xenopus ( $80 \%$ homology) P60-katanin sequences (Fig. 1I). Having obtained the sequence, we prepared a riboprobe to be used for in situ hybridizations and a polyclonal antibody to be used for Western blotting and immunolabeling. P60-katanin, which is a member of the AAA family of ATPases, is a very insol- uble protein, and for this reason, we chose to prepare peptide antibodies as opposed to antibodies against fusion proteins. Our best results were obtained with a peptide region of 45 aa (amino acids 115-148) that shows high homology with the human and mouse sequences (Fig. 1I). The antibody recognizes a single band of the expected mobility on Western blots of RFL6 cells and rat brain (Fig. 1II) as well as a variety of other cells of rodent and human origin (data not shown). In human kidney cell line 293, induced to express a GFP-P60-katanin construct (termed wild type because it has the complete and unaltered sequence of endogenous P60-katanin fused to GFP), a band of the combined molecular weights of P60-katanin and GFP was apparent (data not shown in Fig. 1). We also prepared a construct for expressing a GFP fusion protein for a mutated form of rat P60-katanin in which one amino acid is substituted ( $\mathrm{K}$ to A) in the ATPase domain (Fig. 1I). It has been shown that when the human sequence is mutated in this manner, the resulting construct acts as a dominant-negative that competes with endogenous P60katanin and thereby partially inhibits its activity (McNally et al., 2002). The specificity of the antibody and its suitability for immunolabeling are illustrated in Figure 1, IIA and IIB, which shows low- and high-magnification fields of HeLa cells immunolabeled with the antibody. Interphase cells show diffuse dim staining, whereas cells in various stages of mitosis show staining 

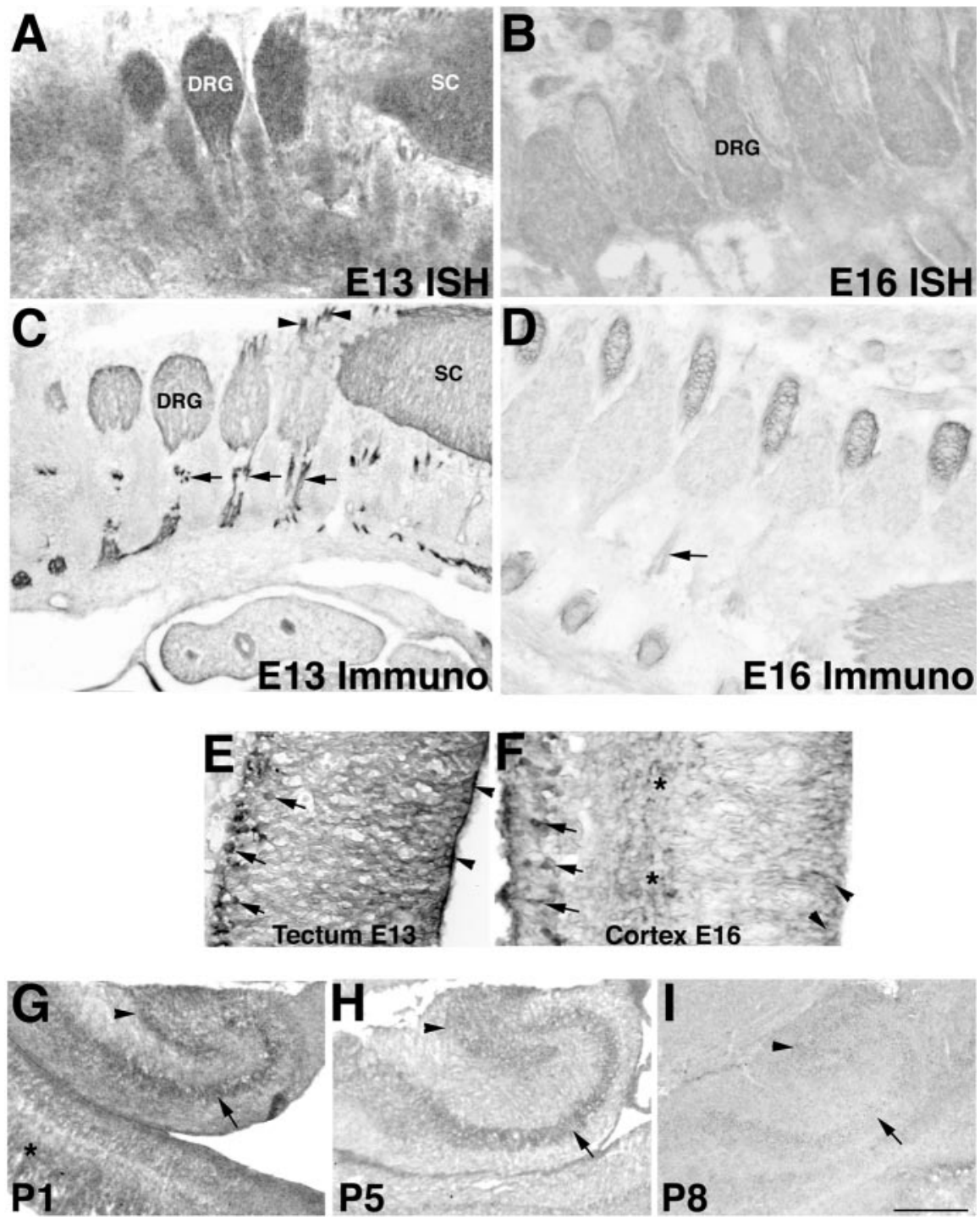

Figure 2. $\quad P 60-$ katanin levels change in peripheral and central neurons during key stages of axonal development. $A, B$, Sections in situ hybridized (ISH) with a riboprobe specific for P60-katanin. DRG, Dorsal root ganglia; SC, spinal cord. P60-katanin mRNA is present in many cell types but is particularly abundant in the developing DRG ( $A$, section from embryonic day 13 mouse). By E16, P60-katanin mRNA levels decline in DRG and surrounding tissues ( $B$, section from an embryonic day 16 mouse). $C-F$, Sections immunolabeled (Immuno) for P60-katanin. C, P60-katanin protein is detected within the DRG but is more abundant in peripheral (arrows) and central (arrowheads) processes of sensory neurons. D, By E16, P60-katanin immunoreactivity is nearly undetectable in the DRG. Low levels of P60-katanin immunoreactivity were detected in spinal nerves containing the peripheral processes of sensory neurons (arrow). Some immunoreactivity also persisted in the spinal cord. E-I, P60-katanin is also expressed and shows changes in expression in the CNS. E, Tectum, embryonic day 13. As shown by immunolabeling, P60-katanin is present in cells and processes near the ventricle (arrowheads) and cells that have migrated toward the pia (arrows). F, Cerebral cortex, embryonic day 16. Labeling was observed in cells adjacent to the ventricle (arrowheads) and their processes. Labeled cells near the ventricle are radial glia or recently born neurons. Immunoreactive cells far from ventricular zones were probably postmigratory neurons of the early cortical plate (arrows). Thalamocortical axons within the cortical intermediate zone are also katanin-immunopositive (asterisks). G-I, P60-katanin expression in the hippocampus is also high in early postmigratory neurons and then declines with additional neuronal maturation. Sections from early postnatal mice in situ hybridized with a riboprobe specific for P60-katanin indicate the developmental changes in the level of P60-katanin. Arrowheads indicate the dentate gyrus, and the arrows indicate Ammon's horn. G, At postnatal day 1 (P1), expression is high in layers containing hippocampal neurons, including the dentate gyrus and Ammon's horn. P60-katanin is also expressed in specific layers of the developing cerebral cortex (asterisk, for example). $H$, By postnatal day 5, low levels of P60-katanin mRNA are present in neuronal layers throughout the hippocampus. I, By postnatal day 8, P60-katanin mRNA levels are very low and near the limits of detection throughout the hippocampus. Scale bar: $A-D, 590$ $\mu \mathrm{m} ; E, F, 100 \mu \mathrm{m} ; G-I, 350 \mu \mathrm{m}$.

of the centrosomes and proximal half-spindles, as accentuated by higher magnification in Figure 1 IIA. Quantification of immunofluorescence indicates that total levels of protein are $18 \%$ higher $(p<0.05$; Student's $t$ test $)$ during mitosis $(19,763 \pm 711 \mathrm{AFU})$ than during interphase $(16,250 \pm 755$ AFU), suggesting that P60-katanin is rapidly synthesized and degraded during the cell cycle (Fig. 1 IIC).

P60-katanin expression changes during development of the nervous system and correlates with axonal growth

As explained in the Introduction, there are strong reasons to believe that katanin activity is determined by the mechanisms that locally activate or deactivate it in response to signals. However, this does not dismiss the possibility that the levels of katanin expression might also be functionally important. To investigate this matter, we performed in situ hybridization and immunolabeling on developing mouse embryos and neonates. On embryonic day 13 (E13), when many regions of the brain are still in early proliferative stages, there was pronounced expression of P60katanin in nervous structures compared with most other regions of the embryo, and expression continued in both the CNS and peripheral nervous system throughout prenatal life. Figure $2 A-D$ shows dorsal root ganglia and their axons, which are components of the peripheral nervous system. At embryonic day 13, in situ hybridization (Fig. 2A) and immunolabeling (Fig. 2C) show that P60-katanin mRNA and protein are particularly high in dorsal root ganglia compared with other cell types. At this point in development, the neurons have already migrated to the ganglia and are in the process of growing their axons. P60-katanin protein was detected in the ganglia themselves but was more abundant in the axons, particularly in the peripheral and central processes of sensory neurons, compared with cell bodies or regions of axons near cell bodies. At embryonic day 16 , by which time most peripheral axonal growth is complete (Bentley and Lee, 2000), P60-katanin expression had dropped to markedly lower levels with regard to both mRNA and protein levels in cell bodies and axons (Fig. $2 B, D$ ).

The CNS also showed expression of P60-katanin that changed during neuronal development. P60-katanin is present in neurons and axons of the embryonic E13 tectum and cerebral cortex near ventricles, in neurons that have migrated toward the pia, and in thalamocortical axons in the intermediate zone (Fig. 2E,F). The tectum levels are down somewhat by day 16 compared with day 13 (data not shown), but not as dramatically as with the dorsal root ganglia, which may relate to the fact that these CNS axons are still growing. Expression remained high in the hippocampus during its early development during embryogenesis (data not shown) but 
showed gradual diminution over the first several postnatal days (Fig. 2G-I). Expression is high in layers containing neurons at postnatal day 1 . By postnatal day 5, low levels of P60-katanin are present throughout the hippocampal neuronal layers, and by postnatal day 8 , the levels are still lower. Thus, in general, katanin levels are elevated during early neuronal development, when axons are forming and growing, but decline at later stages.

To further investigate the potential correlation between axon growth and katanin, we analyzed more thoroughly the developmental changes in P60-katanin levels in specific branches of the fifth cranial nerve, which can be visualized with clarity as they extend toward and reach their targets in the snout. These targets include mechanoreceptors associated with whisker follicles. P60katanin levels were high in axons comprising the nerve on embryonic day 13 , even in axons with tips that had extended to very superficial regions of the dermis (Fig. 3A,B). Many mechanoreceptor targets of these axons are associated with whisker follicles, which are not yet apparent on day 13. By day 16, the levels of P60-katanin were very low, particularly in the distal regions of the axons (Fig. 3E). An intermediate time point, day 14.5, is generally similar to day 13 with regard to P60-katanin levels. However, whisker follicles were first visible on approximately day 14.5 and P60-katanin-expressing axons were associated with these newly formed follicles, suggesting that the diminution occurs quite rapidly after the axonal tips contact their targets. Interestingly, P60katanin immunoreactivity was first lost in the most distal regions of the axon, suggesting a distoproximal diminution of the protein. Together with our observations on other regions of the peripheral nervous system and CNS, these results indicate that P60katanin levels are high within axons during their most active phases of growth but drop once axons have contacted their targets.

\section{Target cells that cause axons to stop growing result in a rapid diminution in katanin levels}

These correlative observations on the rodent nervous system suggest that contact with appropriate targets may cause the rapid changes in P60-katanin levels. To test this experimentally, we used a culture system of neurons from the basilar pontine nuclei. The axons from these neurons stop growing when they reach their major physiological targets, the granule cells of the cerebellum. In culture, the axons also stop growing when they are presented with cerebellar granule cells (Baird et al., 1992; Zhang and Mason, 1998). In cultures grown on laminin in the absence of granule cells, axons showed high levels of P60-katanin immunoreactivity (Fig. $4 B$ ). In distal regions of the axon where P60katanin was particularly high, the microtubule immunofluorescence was low, presumably reflecting enhanced microtubule severing (Fig. 4A). In mixed cultures containing the granule cells, the basilar pontine axons stopped growing as they contacted the target cells and showed dramatically less immunoreactivity for P60-katanin (although microtubule levels remained high) (Fig. $4 C, D)$. We measured katanin immunofluorescence in basilar pontine axons in cultures in which axons and target cell densities were lower to facilitate measurements. P60-katanin levels in axons that had contacted target cells were $60 \%$ lower in their distal region (Fig. $4 E$; see Fig. 4F, $G$ for examples of individual axons). Similar to the observations in brain sections, the diminution in protein in the presence of target cells was less dramatic, but still significant, in more proximal regions of axons where mean katanin immunofluorescence was reduced by $40 \%$ (see Fig. 4 for data; see figure legend for statistical analyses). These results, indicating a significant drop in P60-katanin levels, are reminiscent

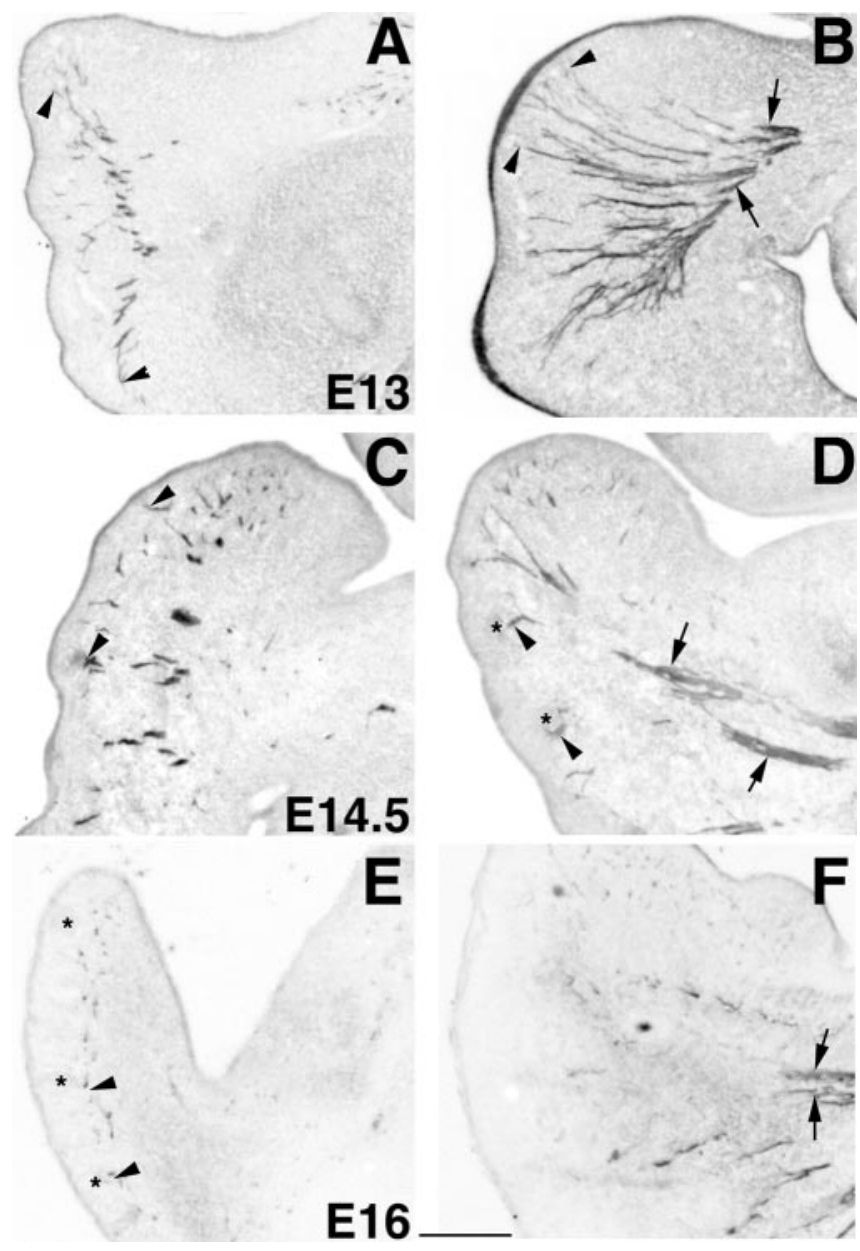

Figure 3. P60-katanin levels change in peripheral axons during development. $A, B$, High levels of P60-katanin were present along the lengths of axons in the fifth cranial nerve on embryonic day 13 , from the distal tips of axons that had extended quite close to where their targets will develop in the snout $(A, B$, arrowheads) to more proximal bundles of axons ( $B$, arrows). Whisker follicles, the locations of many mechanosensory targets of these axons, were not observed at E13. At E14.5, newly formed whisker follicles were present (D, asterisks). P60katanin levels remained high in fifth nerve axons at this time, both in distal tips $(C, D$, arrowheads) and in proximal bundles ( $D$, arrows). By E16, P60-katanin was very low or absent in distal regions of sensory axons. $E$ and $F$ show regions of highest $\mathrm{P} 60$-katanin immunoreactivity in the E16 embryonic snout; little or no immunoreactivity was observed in neighboring sections (data not shown). By E16, immunoreactivity was low and extremely restricted in very distal regions of sensory axons ( $E$, arrowheads) associated with whisker follicles (asterisks). Immunoreactivity was present in more proximal sections of the nerve ( $F$, arrows) but at much lower levels in most axonal regions compared with E13 and E14.5. Scale bar, $240 \mu \mathrm{m}$.

of (but even more dramatic than) the rapid changes observed during the cell cycle (Fig. 1). Measurement of katanin immunofluorescence also supports qualitative observations indicating that katanin levels are generally high distally and somewhat lower more proximally, regardless of whether basilar pontine axons are in contact with cerebellar cells, with immunofluorescence falling on average by $46 \%$ from distal to proximal in axons contacting cerebellar cells and by $30 \%$ in axons not in contact with cerebellar cells (see Fig. 4 and its legend for statistical analyses). It is intriguing that katanin levels are significantly higher in and near growth cones of extending axons and in distal regions where microtubules are generally shorter and more likely to be severed compared with more proximal regions of the axon ( $\mathrm{Yu}$ and Baas, 1994; Dent et al., 1999). 

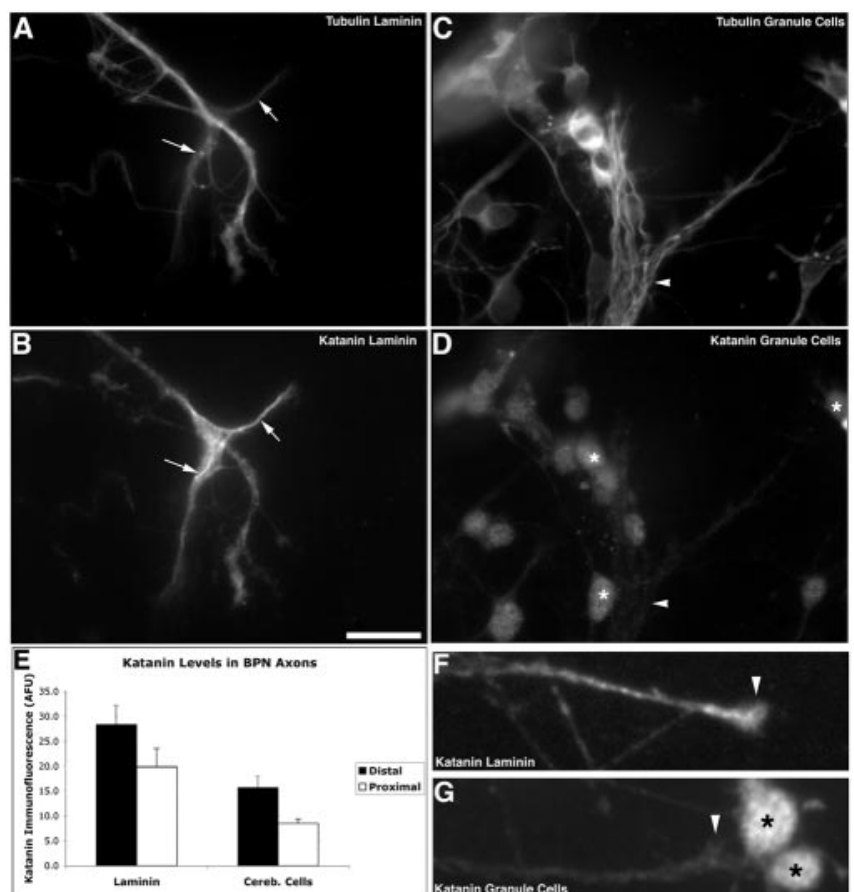

Figure 4. P60-katanin levels decrease within $24 \mathrm{hr}$ after axons encounter target cells. Basilar pontine neurons were grown in culture and double-labeled for $\beta$-tubulin and P60-katanin. In some cultures, the neurons were presented with cerebellar cells, mostly granule neurons, which are a physiological target for the basilar pontine axons; axonal growth ceases as the axons encounter the granule cells. $A$, Axons and growth cones immunolabeled for $\beta$-tubulin after 24 $\mathrm{hr}$ of extension from explants from basilar pontine nuclei explants growing on a laminin substrate in the absence of cerebellar cells. B, Same field as A, labeled for P60-katanin. Regions of the growth cone (shown here is a growth cone that has just recently bifurcated and given rise to two early axonal branches) that are particularly rich in P60-katanin have lower levels of tubulin immunofluorescence, probably reflecting microtubule-severing activity ( $A, B$, arrows). $C$, Basilar pontine axons immunolabeled for $\beta$-tubulin (arrowhead) after $24 \mathrm{hr}$ of coculture with dissociated cerebellar neurons. Cell bodies seen are cerebellar cells, mostly granule cell targets of basilar pontine axons. D, Same field as C, immunolabeled for P60-katanin. Although cerebellar cells often stain brightly for P60-katanin (asterisk, for example), basilar pontine axons have little or no immunoreactivity (arrowhead). E, Mean P60-katanin immunofluorescence in $10 \mu \mathrm{m}$ axon segments at or near distal tips or in $10 \mu \mathrm{m}$ segments located 35-100 $\mu \mathrm{m}$ more proximally within the same groups of axons. Distal and proximal segments were measured in nine axons contacting cerebellar cells (Cereb. Cells) and in nine axons not contacting cerebellar cells (Laminin). Mean katanin levels are significantly reduced in distal regions of basilar pontine axons contacting cerebellar cells, by $60 \%$ at or near growth cones or other types of axonal endings compared with basilar pontine axons not in contact with cerebellar cells in the same cultures $(p<0.03)$. Mean katanin levels in proximal axon segments were significantly reduced by $40 \%$ in basilar pontine axons contacting cerebellar cells compared with axons not contacting target cells in the same culture $(p<0.02)$. Mean katanin levels were in general significantly lower when comparing proximal and distal regions of the same axons regardless of whether they were in contact with cerebellar cells ( $30 \%$ reduction, axons not contacting cerebellar cells, $p<0.03$; $46 \%$ reduction, axons contacting cerebellar cells, $p<0.005$ ). F, Example of high katanin levels in a growth cone (arrowhead) and adjacent distal axon segment in a basilar pontine axon growing on laminin. Katanin levels are reduced in more proximal regions of this axon. G, Example of low katanin levels in a growth cone (arrowhead) in contact with target granule cells (asterisks) in the same culture as the axon in F. Note that katanin levels are also low in more proximal regions of the same axons, whereas katanin levels in the granule neurons are high. Scale bar: $A-D, 25 \mu \mathrm{m} ; F, G, 14 \mu \mathrm{m}$.

Expression of P60-katanin constructs alters the microtubule array of cultured rat sympathetic neurons and affects axonal outgrowth

Although the correlation documented above could be coincidental, it seems likely that it could also reflect a functional relationship between axonal growth and katanin levels. To examine this further, we sought to experimentally manipulate P60-katanin levels to ascertain whether axonal growth is sensitive to such alterations. In our previous study, we documented that complete or nearly complete inhibition of P60-katanin with a functionblocking antibody had dramatic results on cultured rat sympathetic neurons; the microtubules failed to be released from the centrosome, microtubule lengths were increased markedly, and axonal growth was severely compromised (Ahmad et al., 1999). McNally et al. (2002) have established that overexpression of P60-katanin in cultured fibroblasts results in excess severing of microtubules, which in turn results in depolymerization of a portion of the microtubule mass. Here we sought to overexpress P60-katanin in cultured neurons to ascertain how sensitive the neuron is to alterations in katanin levels. We also wanted to lower the levels of active katanin, but in a more subtle manner than in our previous study. For these experiments, we used the wild-type and dominant-negative constructs described previously. Our constructs are based on the rat sequence, but the dominantnegative has the same mutation as the human mutant construct of McNally et al. (2000). Initial studies involved our constructs and those of McNally et al. (2000); we observed entirely similar results with both sets of constructs, confirming the ability of the rat constructs to produce the same results as the proven constructs. One reason for generating rat constructs was to maximize the possibility that the antibody would equally recognize endogenous and expressed P60-katanin, so that we could quantify the levels of expression relative to endogenous levels in nontransfected cells.

Using a culture regimen that has been used previously for studies on axonal growth from sympathetic neurons, we plated the neurons on a polylysine substrate overnight to permit the expressed proteins to accumulate and then treated the cultures with laminin for $8 \mathrm{hr}$ to induce rapid axonal outgrowth (He and Baas, 2003). Under these conditions, control neurons (expressing GFP alone) grew an average of $214 \pm 25 \mu \mathrm{m}$ of axon. GFP expression did not alter the growth properties of the axon compared with nonexpressing neurons in the same culture, but the nucleofection process stunted axonal outgrowth somewhat compared with cultures of neurons that had not undergone the nucleofection process. In terms of P60-katanin levels, control neurons were calculated at 25,193 \pm 835 AFU using the P60katanin antibody. With regard to the P60-katanin constructs, the wild-type construct elevated total P60-katanin levels calculated to $29,278 \pm 1719$ AFU. These calculations indicate a significant difference between the two values ( $p<0.05$; Student's $t$ test), fairly low variation from cell-to-cell, and an average increase in katanin of $16 \%$ above control levels. Figure 5, $A$ and $B$, shows images of P60-katanin immunostaining in a neuron expressing GFP alone (control) and the wild-type construct, showing an increase in fluorescence intensity. Quantitative data are shown in Figure $5 C$. We found that the fluorescence intensity as assessed for GFP correlated fairly well with the staining for P60-katanin in most cells, but this varied somewhat, as expected, because of the normal variability in endogenous katanin levels (data not shown). As expected, the dominant-negative construct expressed equally well as the wild-type construct (data not shown). The average axonal length was $67 \pm 18 \mu \mathrm{m}$ for neurons expressing the dominant-negative construct and $144 \pm 25 \mu \mathrm{m}$ for the neurons expressing the wild-type construct (Fig. $5 D$ ). Thus, there was an overall diminution in axonal length of 69 and $33 \%$, respectively, and these differences were statistically significant $(p<0.001$ and $p<0.05$, respectively; Student's $t$ test). Notably, however, there was no correlation between the levels of expression of the P60katanin constructs (or the total levels of P60-katanin assessed 

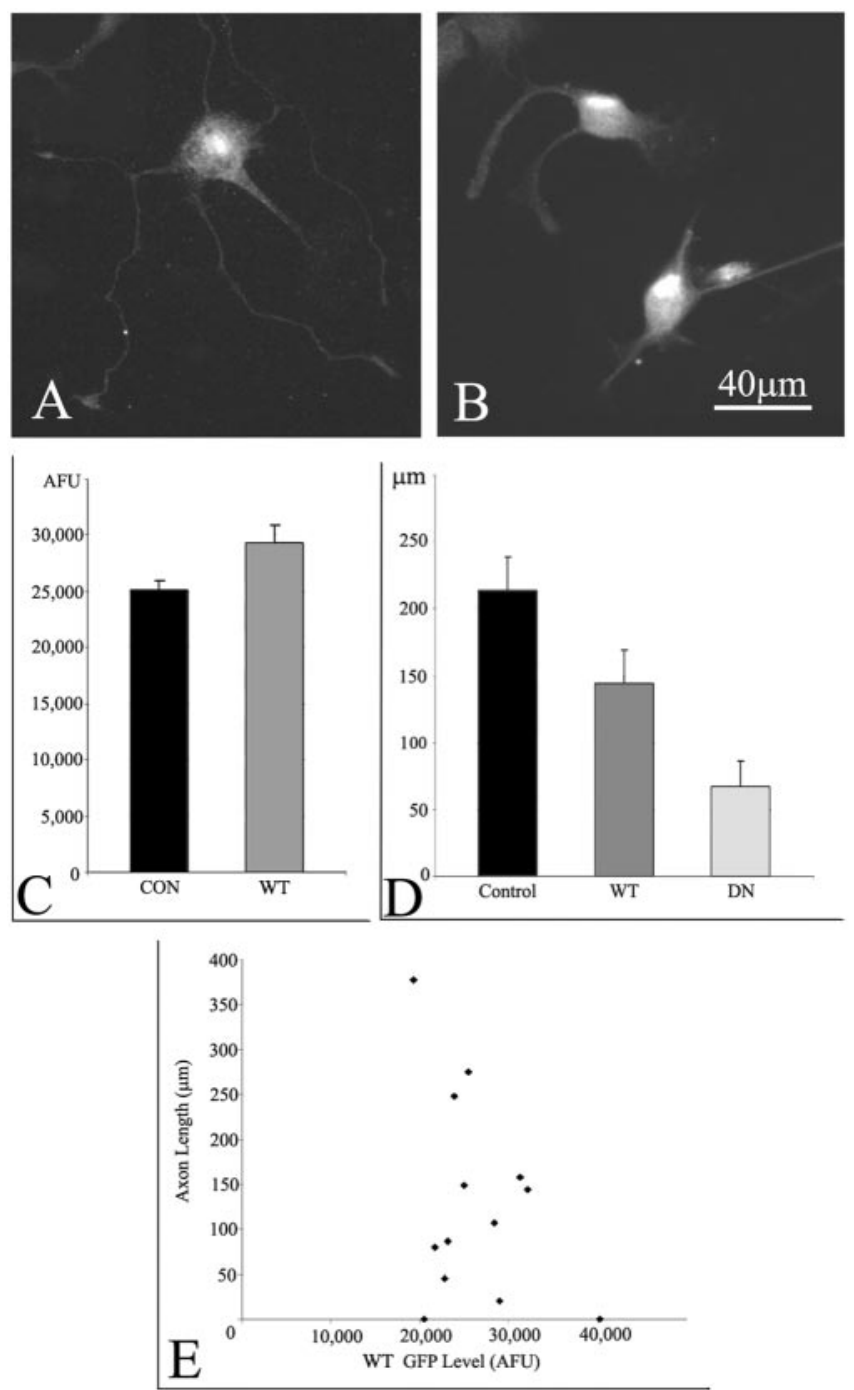

Figure 5. Expression of P60-katanin wild-type and dominant-negative constructs suppresses axonal outgrowth, but not in proportion to the levels of expression. GFP constructs of the wild-type or dominant-negative P60-katanin were transfected into rat sympathetic neurons. A shows immunostaining for P60-katanin in a neuron that was transfected with GFP alone, whereas $B$ shows two neurons that were transfected with the wild-type construct. Axonal outgrowth is less robust in the neurons expressing the wild-type construct. ( shows quantitative data on P60-katanin levels in control (CON) neurons and neurons expressing the wild-type (WT) constructs. The construct raises the total level of immunoreactivity by a mean of $16 \%$, with fairly low variability from neuron to neuron. The dominant-negative construct expressed equally well (data not shown). D shows a marked diminution in axonal length relative to control neurons of neurons expressing the wild-type construct and an even greater diminution in neurons expressing the dominant-negative (DN) construct. $E$ shows axonal length of individual neurons plotted against quantitative data on levels of GFP fluorescence after expression of the wild-type construct. There is no correlation between the amount of expression and the degree to which axonal outgrowth was stunted. A similar lack of correlation was observed with regard to the amount of expression of the dominant-negative and whether we quantified GFP fluorescence or P60-katanin fluorescence. Scale bar, $40 \mu \mathrm{m}$.

with the antibody) and the degree to which axonal length was stunted, and this was true of both the wild-type and the dominant-negative constructs. An example of such an analysis is shown in Figure $5 E$, in which it can be seen that individual neurons expressing very similar levels of the wild-type construct display markedly different axonal lengths.

Morphological features of the neurons are presented in Figure 6 , which shows phase-contrast and GFP images of control neu- rons (expressing GFP alone) (Fig. 6A) and neurons expressing the two constructs. Neurons expressing the dominant-negative construct almost always showed a single short axon with a notable curve. This unipolar morphology has been observed in previous studies on cells in which abnormally long microtubules remain attached to the centrosome, for example after overexpression of MAPs (Avila et al., 1994; Kaech et al., 1996). Some of these short axons displayed an abnormally thickened distal region, which may reflect a distal accumulation of cytoskeletal elements (Fig. 6C). Figure $6 \mathrm{D}$ shows a neuron with no processes at all. Sometimes the axons were short but not curved (Fig. $6 E, F)$. Figure $6 G$ shows a neuron expressing the wild-type P60katanin construct; there is no axonal outgrowth and shrinkage of the cell body. Unlike the case with the dominant-negative construct, when axons were present after expression of the wild-type construct, the axons were typically multiple in number (Fig. $6 H, J, K$ ), but not always (Fig. 6I). In general, the tips of axons were more splayed with the wild-type expression than with the dominant-negative, which is consistent with shorter, more mobile microtubules in the former (Dent et al., 1999). Thus, the morphological observations are consistent with what one might expect of neurons in which microtubule length and attachment to the centrosome have been altered according to the properties of each of the constructs.

In another set of experiments, we used an experimental regimen that revealed more complexity in the response of the neurons to katanin manipulation, in particular to the wild-type construct. In these experiments, we expressed the constructs in sympathetic neurons and plated them very densely for $2 \mathrm{~d}$ on plastic. Neuronal development in these sympathetic cultures is known to be very density dependent (He and Baas, 2003), and we reasoned that growing them in this manner might cause them to express more highly an abundance of other microtubuleregulatory proteins that are potentially relevant to katanin activity. This is supported by the fact that outgrowth of control neurons was far more robust, with average lengths of $645 \pm 73 \mu \mathrm{m}$ of axon per neuron. After $2 \mathrm{~d}$, the neurons were replated on a polylysine-laminin substrate and axonal growth was quantified. (After replating there was also no evidence of any residual ill effects of the nucleofection process, with GFP-only nucleofected neurons showing indistinguishable levels of outgrowth compared with similarly treated cultures from neurons that had not undergone nucleofection.) The data are presented in Figure 7. On average, axonal outgrowth was reduced markedly with the wildtype construct (to $253 \pm 88 \mu \mathrm{m}$ ) and even more so with the dominant-negative (to $101 \pm 34 \mu \mathrm{m}$ ), as was the case with the first experimental regimen. Here, the reductions compared with the controls were 61 and $84 \%$, respectively. The most notable difference between this experimental regimen and the other regimen was that approximately one-half of the neurons expressing either the dominant-negative or the wild-type construct failed to grow any axons at all in the replating regimen, whereas this was rarely observed in the neurons expressing either of the constructs in the first experimental regimen. When only process-bearing neurons were included in the statistical analyses, the effect with the dominant-negative was still a $69 \%$ diminution in axonal growth compared with controls (with an average axonal length of $201 \pm 32 \mu \mathrm{m})$. In contrast, the effect with the wild-type construct was more "all-or-nothing," with the process-bearing neurons not showing any consistent morphological differences or any statistically significant difference ( $p>0.05$; Student's $t$ test) in mean axonal length compared with the controls between the two populations (with an average axonal length for the process-bearing 


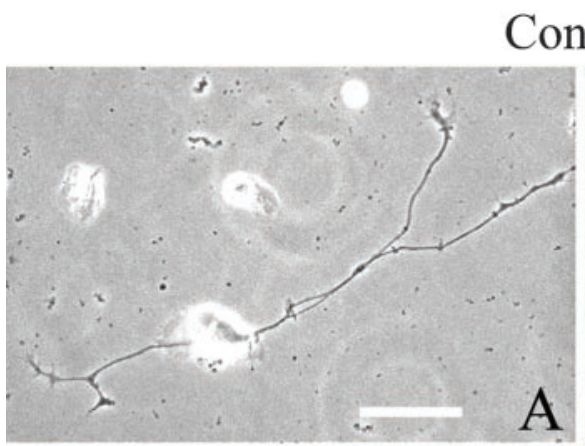

Control

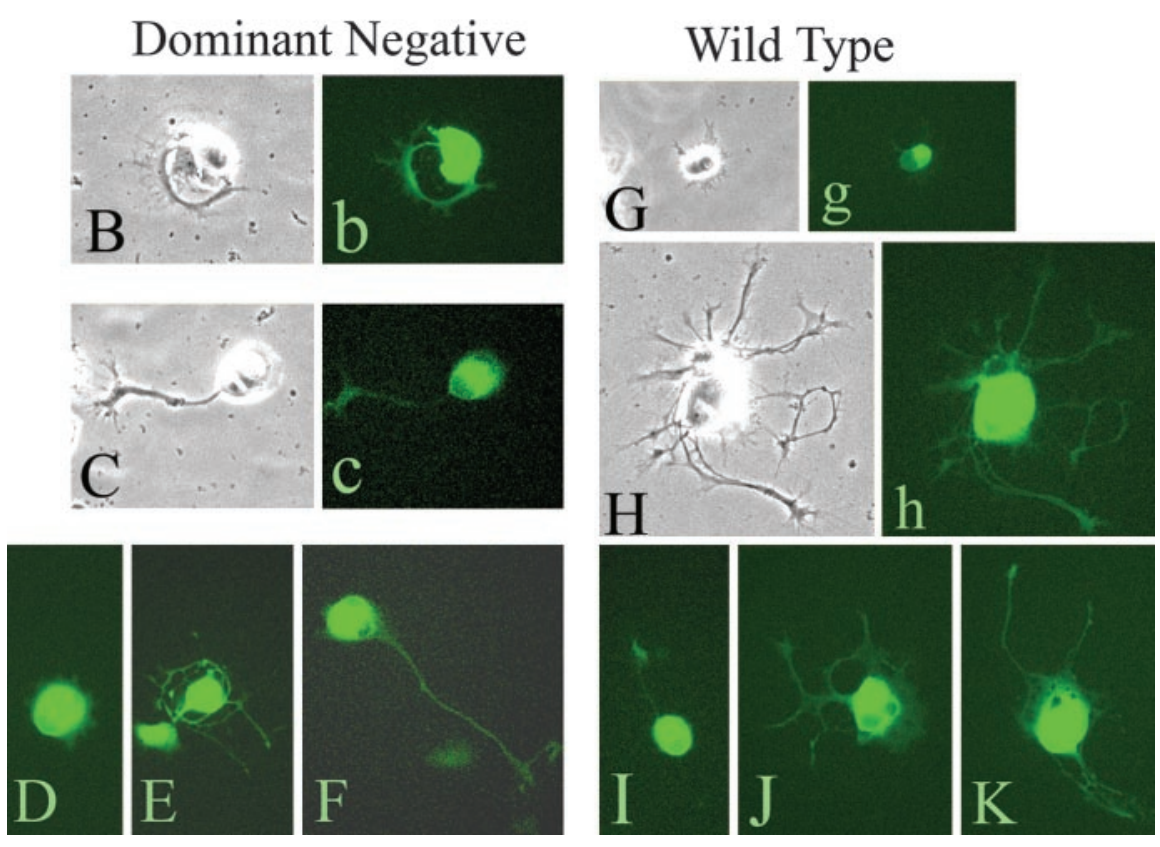

Figure 6. Morphological features differ in neurons expressing either the P60-katanin wild-type or a dominant-negative construct. Phase-contrast and fluorescence images of the transfected neurons after $8 \mathrm{hr}$ of axonal outgrowth are shown. The neurons were still alive at the time the images were acquired. Fluorescence images are of GFP and are not augmented with antibody staining to enhance the intensity. In A, GFP alone is expressed in control neurons; expression does not alter the growth properties of the axon compared with nonexpressing neurons. Neurons expressing the dominant-negative construct often show a single short axon with a notable curve $(B)$ or a single short axon with an abnormally thickened distal region (C.D-Fshow three other examples of neurons expressing the dominantnegative construct showing other morphologies. Neurons with no outgrowth at all were rare (an example is in shown in D), except with the replating experimental regimen (see Results). Neurons expressing the wild-type P60-katanin construct also rarely showed no axonal outgrowth at all (except in the replating regimen) but showed a notable reduction in size on the rare occasion when there was no outgrowth ( $G$ ). Neurons expressing the wild-type P60-katanin construct typically showed short axons, often multiple in number $(H)$. $I-K$ show three other examples of neurons expressing the wild-type construct with varying degrees of axonal outgrowth. Neurons shown in both phase-contrast and fluorescence are labeled with capital and small letters, respectively, whereas neurons shown only in fluorescence are labeled with capital letters. Scale bar, $25 \mu \mathrm{m}$.

wild-type expressers of $506 \pm 122 \mu \mathrm{m})$. However, there was a great deal of variability among the individual neurons expressing the wild-type construct (as evidenced by the high SEM), with some of them actually displaying longer axons than any of the neurons in the control group. Figure $7 A$ shows a typical control neuron, whereas Figure $7 B$ shows two neurons expressing the wild-type P60 construct. One of these neurons shows no axons at all, whereas the other displays a longer axonal arbor than any of the control neurons examined in the same experiment. These results demonstrate that individual neurons can react very differently to experimental increases in P60-katanin levels.

To confirm that the constructs were having the expected effects on the microtubule array, we immunostained the cultures to visualize microtubules and then examined the cells with confocal microscopy. Optical sections were $0.2 \mu \mathrm{m}$ in thickness, and com-

\section{Discussion}

parisons were made between sections taken at similar planes in different cells to compare microtubule length and distribution. Length comparisons are qualitative only because individual microtubules (particularly longer ones) may appear on more than one optical section. Both neurons and fibroblastic cells in the cultures were transfected. As expected, the wild type diminished microtubule levels, whereas the dominant-negative caused accumulations of microtubules, particularly in the centrosomal region. Figures 8 and 9 show some of the more dramatic examples of fibroblasts and neurons in which the alterations in the microtubule array are particularly clear. Figure $8 A$ shows a control fibroblast with a typical array of splayed microtubules emanating from the centrosome, whereas Figure $8 D$ shows a neuron in the region of the cell body with a typical dense scattered microtubule array. Figure $8, B$ and $E$, shows optical sections at the plane of the centrosome after expression of the dominant-negative construct in a fibroblast and neuron, respectively. Microtubule accumulations in the centrosomal region are apparent. Figure $8, C$ and $F$, shows optical sections below the plane of the centrosome in a fibroblast and neuron, respectively. The microtubule profiles are generally longer than in control cells. Figure 9, $A$ and $B$, shows fibroblasts expressing the wild-type P60-katanin construct; microtubule levels are lower than in controls, and the central region of the cells is sparse in microtubules compared with controls. Many very short microtubules are apparent (as expected), but there are also some longer microtubules, suggesting that certain regions of longer microtubules may be less susceptible to breakage by katanin. Similar results were obtained on neurons (Fig. 9C-E). Figure $9 E$ is an optical section below the nucleus showing that not all microtubules scatter to the cell periphery. In many cells showing such severe effects on the microtubules, the cell bodies tended to shrink in size. Neurons expressing the wild-type construct, which failed to show stunted axonal growth (see above), also failed to show any detectable alterations in their microtubule arrays compared with control neurons (data not shown in Fig. 9). Together, these results are consistent with an enhancement of microtubule severing by the wild-type construct and a partial inhibition of severing by the dominant-negative construct but demonstrate that the microtubules within some neurons are notably less responsive to experimental increases in P60-katanin levels than others.

Our first indications that microtubules are severed in neurons came from studies demonstrating that microtubules are nucleated exclusively at the centrosome, and hence must be severed 
from the centrosome for transport into the axon (Baas and Joshi, 1992; Yu et al., 1993; Ahmad et al., 1994). A centrosomal origin for axonal microtubules begged the question of how microtubule numbers are increased at sites distal to the centrosome, such as during the formation of collateral axonal branches. We speculated that long microtubules in the axon might be severed into multiple shorter microtubules at strategic locations such as branch points (Joshi and Baas, 1993). This speculation was borne out by the results of serial reconstruction analyses of electron micrographs of axons undergoing branch formation (Yu et al., 1994). Since these early studies, we have directly visualized the breakage of microtubules at axonal branch points and also within growth cones that have begun to move after having been stalled (Dent et al., 1999). These observations, together with the widespread distribution of katanin in the neuron (Ahmad et al., 1999), have led to the conclusion that microtubule severing by katanin is an important phenomenon for regulating microtubule length and number throughout the neuron, as well as for releasing microtubules from the centrosome. The importance of microtubule severing is also accentuated by studies suggesting that the capacity of a microtubule to be transported is inversely proportional to its length (Dent et al., 1999; Wang and Brown, 2002).

In the present study, we observed some enrichment of katanin in the more distal region of the axon, where one might expect microtubule severing to be more common. However, katanin is also present virtually everywhere in the neuron, suggesting that cells must be able to strategically regulate the severing activity of katanin so that microtubules are not broken into smaller pieces everywhere at all times. Indeed, studies in vitro have shown that katanin, if constitutively active, would continue to sever microtubules completely into subunits (McNally and Vale, 1993; Davis et al., 2002). Studies on cellular extracts indicate that the severing of microtubules by katanin occurs more avidly during mitosis than during interphase, and that this difference somehow relates to phosphorylation (Vale, 1991). Interestingly, however, there is no evidence from other cell types that katanin itself is phosphorylated (McNally et al., 2002). Although this remains to be tested in neurons, it seems reasonable that katanin might be regulated by the phosphorylation of other proteins. Microtubules break in fibroblasts after only slight bending (Gumpton et al., 2002), whereas microtubules do not break after severe bending and contortion in purified microtubule preparations (Janson et al., 2003) and within axons undergoing retraction ( $\mathrm{He}$ et al., 2002). We suspect that bending alone does not cause microtubules to break in the absence of katanin, but that when katanin is present, bending can lead to breakage because it enhances the access of katanin to the lattice (Odde et al., 1999). Compared with fibroblasts, neurons contain a far richer array of MAPs that could protect the microtubule from being accessed by katanin. Consistent with this view, the association of many MAPs with the microtubule is known to be regulated by phosphorylation, which can be altered locally by signaling cascades (Avila et al., 1994; Ebneth et al., 1999). Although it is not yet clear whether MAPs bind at sufficient levels to block access of katanin, this model is attractive
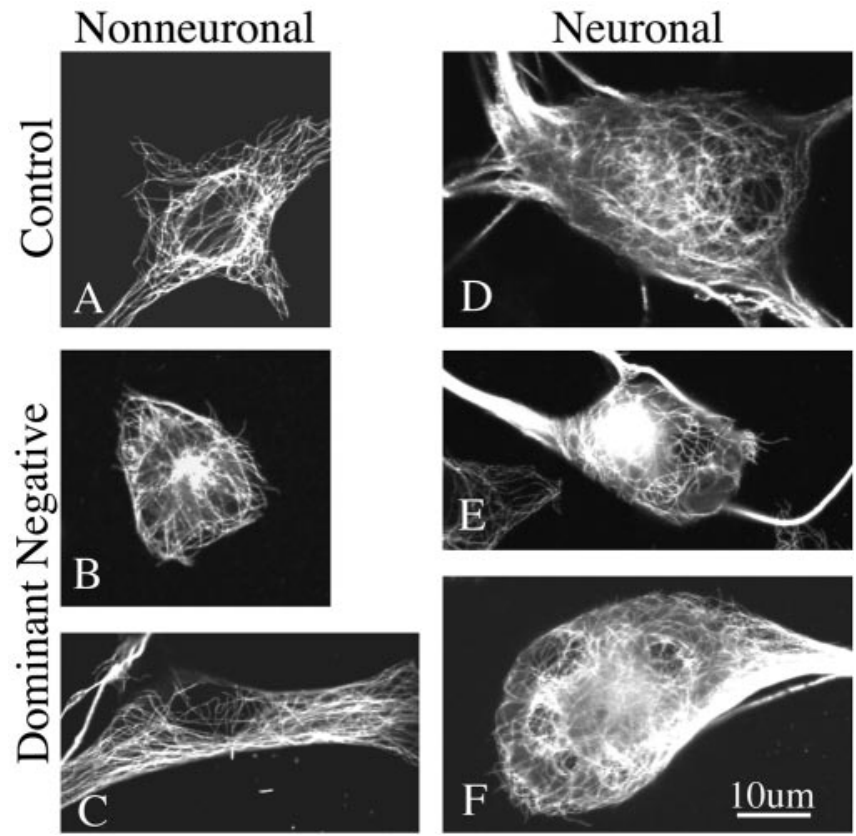

Figure 8. Expression of the P60-katanin dominant-negative construct alters the microtubule array of cultured neurons. Both non-neuronal fibroblastic cells $(A-C)$ and neurons $(D-F)$ that exist within the cultures derived from rat sympathetic ganglia are shown. 0 ptical sections were taken at different planes. Control non-neuronal cells show a typical radial array of microtubules emanating from a centrosomal region $(A)$. Non-neuronal cells overexpressing the dominant-negative construct show an abnormal accumulation of microtubules, reflecting the expected partial inhibition of release ( $B$, on a plane that accentuates the centrosome) and an apparent increased microtubule length compared with control cells $(C$, on a plane below the centrosome). A control neuron is shown in $D$, and an abnormal accumulation of microtubules, reflecting the expected partial inhibition of release, is also observed in neurons overexpressing the dominant-negative construct ( $E$, on a plane that accentuates the centrosome). Neurons overexpressing the dominant-negative construct also showed an apparent increased microtubule length compared with control cells ( $F$, on a plane below the centrosome). Note that the increases in microtubule length are only representative of alterations in microtubule length, because individual microtubules may traverse more than one optical section. Scale bar: $A-F, 10 \mu \mathrm{m}$. 


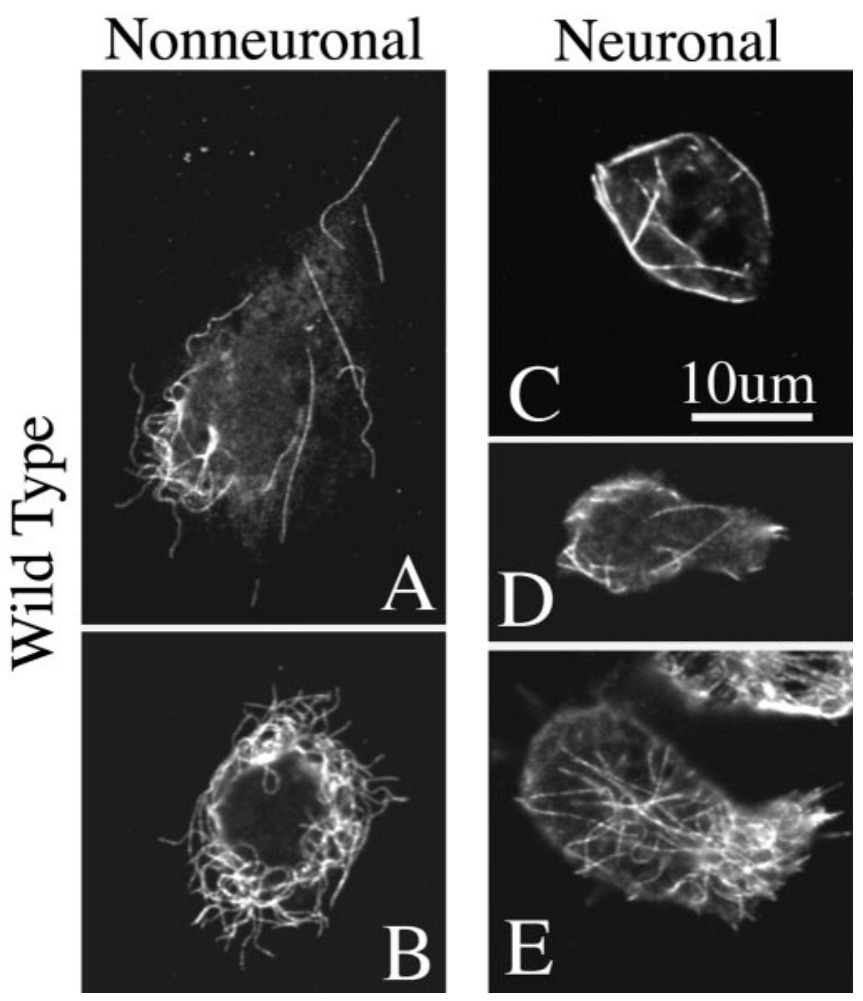

Figure 9. Expression of the P60-katanin wild-type construct alters the microtubule array of cultured neurons. Both non-neuronal fibroblastic cells $(A, B)$ and neurons $(C-E)$ that exist within the cultures derived from rat sympathetic ganglia are shown. Optical sections were taken at various planes. Non-neuronal cells expressing the construct show an absence of microtubules from the central region of the cell body, an overall diminution in total microtubule levels, and a notable abundance of very short microtubules. Microtubules several micrometers in length were also present $(A)$, suggesting that some regions of the microtubules may be less susceptible to severing by katanin, perhaps because of microtubule-associated proteins that block access to the lattice. Neurons expressing the construct show comparable results $(C-E)$. E is at a plane below the nucleus against the culture dish, revealing that not all microtubules scatter to the cell periphery after enhanced katanin-induced severing. Scale bar: $A-E, 10 \mu \mathrm{m}$.

because it provides a means by which microtubule severing could be tightly and focally regulated.

With all of this in mind, the overall goal of the present study was to ascertain whether katanin levels vary during neuronal development and, if so, how responsive neurons are to such fluctuations. We found that katanin levels do in fact vary substantially during neuronal development and that the levels of katanin correlate very nicely with the state of axonal growth. In both immunolabeling and in situ hybridization analyses on the developing CNS and peripheral nervous system, we observed marked differences in P60-katanin levels in different kinds of neurons and at different stages of development. The most interesting result was that the protein levels were comparatively high during phases of rapid axonal growth but then dropped rapidly when the axon reached its target and stopped growing. To investigate this correlation experimentally, we examined P60-katanin levels in cultured neurons allowed to grow axons avidly versus axons that were presented with target cells that induce them to stop growing. Consistent with the observations on the intact animals, the levels of P60-katanin were high in the rapidly growing axons but dropped rapidly once the target cells were introduced. These observations are reminiscent but even more dramatic than those on dividing cells, in which we observed a diminution in P60-katanin during the transition from mitosis to interphase. Thus it appears that cells have the capacity to rapidly synthesize and degrade
P60-katanin in response to both transitions in the cell cycle and external cues relevant to axonal development. Degradation of many proteins is regulated during the cell cycle by signaling mechanisms involving events such as ubiquitination. It will be of interest to elucidate potential signals for katanin degradation during the cell cycle and in neurons. Notably, the higher katanin levels correspond to phases during which we would expect microtubule severing to be more active, given that microtubule release from the centrosome is more active during mitosis than during interphase and that shorter microtubules are expected to move faster, which would augment axonal growth.

These results indicate that alterations in the levels of katanin could be quite relevant to its function in the nervous system, despite the fact that local mechanisms are also important for its regulation. To study this further, we manipulated katanin in cultured neurons and ascertained the effects on the microtubule array and axonal development. In our previous study, we showed that microinjection of a function-blocking antibody against P60katanin causes microtubules to remain attached to the centrosome and increases the lengths of the microtubules within the cell body (Ahmad et al., 1999). These effects were deleterious to axonal growth, which is not surprising given that the experiments were designed to maximally inhibit katanin. Here we wanted to investigate the sensitivity of the neuron to more subtle changes in katanin levels. Expression of the dominant-negative rat P60katanin construct yielded results consistent with those obtained with the function-blocking antibody; axonal growth was stunted, and the microtubule array displayed virtually the same alterations as with the antibody, although the alterations were somewhat less dramatic. Expression of the wild-type construct also stunted axonal growth in most neurons, but these results were more variable if the neurons were replated after expressing the construct for $2 \mathrm{~d}$. Under these conditions, half of the neurons displayed no outgrowth at all, whereas the other half displayed highly variable levels of outgrowth, with some extending axons longer than observed in any of the control neurons. In the cases in which axonal outgrowth was stunted or completely obliterated, there were notable effects on the microtubule array, with significant diminutions in total microtubule mass as well as the appearance of shorter microtubules than observed in control neurons. No such effects were observed in the neurons expressing the wildtype construct that grew axons similar to or longer than those extended by control neurons. There was no correlation among the levels of P60-katanin, whether the neuron grew axons, or the length of the axons. These results indicate that an elevation in P60-katanin can elicit extremely dramatic changes in the microtubule array or can elicit virtually no detectable change at all. In the latter case, there may be changes that are too subtle to detect by our methods, such as a selective shortening of a fraction of the microtubule array, which might augment axonal outgrowth by speeding the average transport velocity of microtubules underlying the growth of the axon (Wang and Brown, 2002). This would be consistent with our observations of higher levels of P60katanin in axons during their most active phases of growth during normal development.

We suspect that the variability from neuron to neuron relates to the fact that katanin is regulated by mechanisms other than its absolute levels, as discussed previously. For example, the levels and disposition of certain MAPs might provide a buffer against sudden increases in katanin levels, and this might vary in different neurons depending on a variety of intrinsic and extrinsic factors. Katanin might also act synergistically with other proteins that destabilize or sever microtubules, such as stathmin (Grenningloh 
et al., 2004), spastin (Errico et al., 2002), or kinesin superfamily protein 2A (Homma et al., 2003). Another possibility is that P60katanin is regulated by some aspect of P80-katanin that is not yet clear. Current efforts in our laboratory are focused on testing potential roles for MAPs, P80-katanin, and other cytoskeletal proteins in regulating the severing properties of $\mathrm{P} 60$-katanin. On the basis of the present results, we find it provocative to speculate that the mechanisms that control katanin levels and activity could be key to determining the growth properties of the axon.

\section{References}

Ahmad FJ, Joshi HC, Centonze VE, Baas PW (1994) Inhibition of microtubule nucleation at the neuronal centrosome compromises axon growth. Neuron 12:271-280.

Ahmad FJ, Echeverri CJ, Vallee RB, Baas PW (1998) Cytoplasmic dynein and dynactin are required for the transport of microtubules into the axon. J Cell Biol 140:246-256.

Ahmad FJ, Yu W, McNally FJ, Baas PW (1999) An essential role for katanin in severing microtubules in the neuron. J Cell Biol 145:305-315.

Ahmad FJ, Hughey J, Wittmann T, Hyman A, Greaser M, Baas PW (2000) Motor proteins regulate force interactions between microtubules and microfilaments in the axon. Nat Cell Biol 2:276-280.

Avila J, Dominguez J, Diaz-Nido J (1994) Regulation of microtubule dynamics by microtubule-associated protein expression and phosphorylation during neuronal development. Int J Dev Biol 38:13-25.

Baas PW, Black MM (1990) Individual microtubules in the axon consists of domains that differ in both composition and stability. J Cell Biol 115:1333-1344.

Baas PW, Buster DW (2004) Slow axonal transport and the genesis of neuronal morphology. J Neurobiol 58:3-17.

Baas PW, Joshi HC (1992) Gamma-tubulin distribution in the neuron: implications for the origins of neuritic microtubules. J Cell Biol 119:171-178.

Baird DH, Hatten ME, Mason CA (1992) Cerebellar target neurons provide a stop-signal for afferent neurite extension. J Neurosci 12:619-634.

Baird DH, Hatten ME, Heintz N, Mason CA (1994) Micro-methods for analyzing axon-target interactions in vitro. NeuroProtocols: a Comp to Meth in Neurosci 4:106-115.

Bentley CA, Lee KF (2000) p75 is important for axon growth and Schwann cell migration during development. J Neurosci 20:7706-7715.

Davis LJ, Odde DJ, Block SM, Gross SP (2002) The importance of lattice defects in katanin-mediated microtubule severing in vitro. Biophys J 82:2916-2927.

Dent EW, Callaway JL, Szebenyi G, Baas PW, Kalil K (1999) Reorganization and movement of microtubules in growth cones and developing interstitial branches. J Neurosci 9:8894-8904.

Ebneth A, Drewes G, Mandelkow EM, Mandelkow E (1999) Phosphorylation of MAP2c and MAP4 by MARK kinases leads to the destabilization of microtubules in cells. Cell Motil Cytoskeleton 44:209-224.

Errico A, Ballabio A, Rugarli EI (2002) Spastin, the protein mutated in autosomal dominant hereditary spastic paraplegia, is involved in microtubule dynamics. Hum Mol Genet 11:153-163.

Grenningloh G, Soehrman S, Bondallaz P, Ruchti E, Cadas H (2004) Role of the microtubule destabilizing proteins SCG10 and stathmin in neuronal growth. J Neurobiol 58:60-69.

Gumpton SL, Salmon WC, Waterman-Storer CM (2002) Converging populations of f-actin promote breakage of associated microtubules to spatially regulate microtubule turnover in migrating cells. Curr Biol 12:1891-1899.

Hartman JJ, Mahr J, McNally K, Okawa K, Iwamatsu A, Thomas S, Cheesman J, Heuser J, Vale RD, McNally FJ (1998) Katanin, a microtubulesevering protein, is a novel AAA ATPase that targets to the centrosome using a WD40-containing subunit. Cell 93:277-287.

Hatten ME, Gao WQ, Morrisson ME, Mason CA (1998) The cerebellum: purification and coculture of identified cell populations. In: Culturing nerve cells (Banker G, Goslin K, eds), pp 419-459. Cambridge, MA: MIT

He Y, Baas PW (2003) Growing and working with peripheral neurons. Methods Cell Biol 71:17-35.

He Y, Yu W, Baas PW (2002) Microtubule reconfiguration during axonal retraction induced by nitric oxide. J Neurosci 22:5982-5991.

Homma N, Takei Y, Tanaka Y, Nakata T, Terada S, Kikkawa M, Noda Y, Hirokawa N (2003) Kinesin superfamily protein 2A (KIF2A) functions in suppression of collateral branch extension. Cell 114:229-239.

Janson ME, de Dood ME, Dogterom M (2003) Dynamic instability of microtubules is regulated by force. J Cell Biol 161:1029-1034.

Joshi HC, Baas PW (1993) A new perspective on microtubules and axon growth. J Cell Biol 121:1191-1196.

Kaech S, Ludin B, Matus A (1996) Cytoskeletal plasticity in cells expressing neuronal microtubule-associated proteins. Neuron 17:1189-1199.

McNally FJ, Vale RD (1993) Identification of katanin, an ATPase that severs and disassembles stable microtubules. Cell 75:419-429.

McNally KP, Bazirgan OA, McNally FJ (2000) Two domains of p80 katanin regulate microtubule severing and spindle pole targeting by $\mathrm{P} 60$ katanin. J Cell Sci 113:1623-1633.

McNally KP, Buster D, McNally FJ (2002) Katanin-mediated microtubule severing can be regulated by multiple mechanisms. Cell Motil Cytoskeleton 53:337-349.

Odde DJ, Ma L, Briggs AH, DeMarco A, Kirschner MW (1999) Microtubule bending and breaking in living fibroblast cells. J Cell Sci 112:3283-3288.

Quarmby LM, Lohret TA (1999) Microtubule severing. Cell Motil Cytoskeleton $43: 1-9$.

Sambrook J, Fritsch EF, Maniatis T (1989) Molecular cloning. A laboratory manual, Ed 2. Cold Spring Harbor, NY: Cold Spring Harbor Laboratory.

Solowska JM, Mazurek A, Weinberger L, Baird DH (2002) Pontocerebellar axon guidance: neuropilin-1 and Sema3A-sensitivity gradients across basilar pontine nuclei, Sema3A variation across cerebellum. Mol Cell Neurosci 21:266-284.

Vale RD (1991) Severing of stable microtubules by a mitotically activated protein in Xenopus egg extracts. Cell 64:827-839.

Wang L, Brown A (2002) Rapid movement of microtubules in axons. Curr Biol 12:1496-1501.

Yu W, Centonze VE, Ahmad FJ, Baas PW (1993) Microtubule nucleation and release from the neuronal centrosome. J Cell Biol 122:349-359.

Yu W, Ahmad FJ, Baas PW (1994) Microtubule fragmentation and partitioning in the axon during collateral branch formation. J Neurosci 14:5872-5884.

Zhang Q, Mason CA (1998) Developmental regulation of mossy fiber afferent interactions with target granule cells. Dev Biol 195:75-87. 